\title{
EN DIRECTO, EN VIVO EL ROCK TELEVISADO EN LATINOAMÉRICA ENTRE 1960 Y 1990
}

\author{
LIVE \\ THE TELEVISED ROCK IN LATIN AMERICA BETWEEN 1960 AND 1990 \\ María Paula Cannova \\ mpcgalactica@gmail.com \\ Facultad de Bellas Artes. Universidad Nacional de La Plata. Argentina
}

Recibido: 14/12/2017 | Aceptado: 8/4/2018

\section{RESUMEN}

En este artículo se estudia la reformulación que la televisión latinoamericana en directo produce en los conceptos operatorios del rock de la región, como música popular, a través del marco teórico aportado por el materialismo filosófico respecto de la televisión formal (Bueno, 2000). se analizan las transformaciones que de algunos conceptos musicales hacen músicos y realizadores audiovisuales a partir de su interacción en la transmisión televisiva, a la vez que se contemplan los vínculos con la industria fonográfica multinacional. se consideran programas de la televisión argentina y brasileña como muestras de las unidades de análisis.

\section{PALABRAS CLAVE}

Rock; Latinoamérica; televisión; industria fonográfica

\section{ABSTRACT}

In this article we study the reformulation that live Latin American television produces in the operative concepts of rock in the region, as urban popular music, based on the theoretical framework provided by philosophical materialism with respect to formal television (Bueno, 2000). We analyzed the transformations of some musical concepts made by musicians and audiovisual producers from their interaction in the television broadcast. We also consider the links with the multinational phonographic industry. Argentine and Brazilian television programs are considered as samples of the analysis units.

\section{KEYWORDS}

Rock; Latin America; television; phonographic industry 
A diferencia de la radio, donde el hecho de la transmisión se indica con la fórmula en el aire, la televisión expone esa situación con la enunciación: en directo. La transmisión en directo o televisión formal (Bueno, 2000) se diferencia de la diferida -televisión material- y de la proyección cinematográfica. No obstante, el vínculo cultural, comercial y técnico de la televisión con la radio ha sido permanente, aunque históricamente negado (Pérez Ornia, 2011, p. 18). La música formó parte de la radio y de la televisión desde sus orígenes, siendo realizada in situ o transmitida por ellas. El certamen o concurso musical ha estado presente en la programación radial y televisiva desde sus inicios, incorporando jurados especializados y/o selecciones del público. De igual forma, la presentación en vivo de números musicales conformó gran parte de los contenidos tanto de la radio como de la televisión. Por consiguiente, la música constituye uno de los elementos narrativos, compositivos y disciplinares de ambos medios. Asimismo, la industria musical utiliza dichos medios para la promoción y la difusión de sus productos y artistas. Como ejemplo podemos mencionar a Discodromo Show, el programa televisivo que desde diciembre de 1962 se transmitió por canal Tele 12 de Uruguay. El programa, conducido y producido por Rubén Castillo, poseía su antecedente homónimo diario en radio Sarandí. La versión televisiva incluía una orquesta en vivo, escenografías adaptadas a los artistas y una diversidad de géneros musicales (melódico, beot, rock, candombe beat, canto popular uruguayo, folklore tradicional, tango y tropical). Constituía una plataforma de lanzamiento para los artistas de sellos discográficos uruguayos como Sondor y fue el espacio televisivo que permitió el ingreso del rock y el beat en la pantalla uruguaya con grupos como Psiglo, El Kinto, los Delfines y los Killers, entre otros.

El filósofo español Gustavo Bueno (2000) argumentó sobre la capacidad para reformular los conceptos de verdad y apariencia que la televisación en directo posee mediante la experiencia televidente, es decir, la de hacer posible la visualización de objetos a través de cuerpos opacos. Es en su dimensión dramática, en el sentido de posibilitar la experiencia similar a quien está en el lugar de los hechos, donde la reformulación adquiere la capacidad de transformación de los conceptos operatorios. En tal proceso los conceptos operatorios que explican un determinado tema son redefinidos en su expresión verbal o en su dimensión realizativa.

La programación televisiva de música en vivo presenta diferencias con los documentales televisivos. En primer orden, debido a su situación en tiempo real y, en segundo, a causa de las condiciones de impacto en las audiencias o mediciones de rating. La imposibilidad del control de la edición y las orientaciones de un programa en función de la audiencia medida disponen condiciones particulares para la televisación en directo. Si bien el directo realizado (Bourdon, 1997) permite considerar cercanías en el efecto documentalizante, la televisión formal puede transfigurar los conceptos operatorios (Bueno, 2000) en su dimensión enunciativa y performática. 
Es en esa intersección donde realizadores, audiencia y género cultural se interrelacionan y dotan a esa praxis de una potencia transformadora. Otro aspecto que diferencia al documental sobre música es su funcionamiento como dispositivo de difusión y de legitimación de las temáticas musicales incluidas. Por ejemplo, a diferencia del rockumentory, donde son validados estilos sonoros y momentos históricos «[...] como algo digno de más seria atención» (Shuker, [1994] 2001, p. 180), la televisación de eventos musicales no ha encontrado argumentos tan nobles como la seriedad o su aporte al conocimiento del campo musical.

Se considera aquí que el estudio de la televisación en directo de la música en vivo puede aportar a la comprensión de la reformulación conceptual que la televisión en directo produce. Esta transformación no sólo opera en un nivel enunciativo sino que también lo hace mediante el propio tratamiento audiovisual, reforzando, cuestionando o anticipando dimensiones significativas del campo musical. Tal situación incide en la capacidad para exponer con términos específicos aspectos propios de la música que resignifican las ideas de verdad y apariencia, entendidas como correlativas. Dicho tratamiento audiovisual implica modificaciones a la forma de presentación que buscan conscientemente acercar la experiencia del estudio de televisión al telespectador, tal y como lo expone el sonidista Zuza Homen de Mello (2003), quien indica haber colocado un micrófono suspendido en el techo del teatro desde donde se transmitía el festival a los fines de captar la reacción del público en el estudio y disponerla a los telespectadores para buscar empatía.

A los fines de circunscribir el objeto de estudio, se considerarán dos casos comprendidos a partir de la existencia de video tope, con acceso a los archivos, de fuentes primarias, donde se constata la transmisión efectuada en dos programas de canales abiertos de la televisión argentina y brasileña. En tales muestras de la investigación se buscaron relaciones entre una consideración formal del género rock y las ampliaciones significativas a partir de los usos socioculturales del mismo en el entramado del comercio multinacional fonográfico y mediático. Esto implicó comprender al género musical como una unidad dinámica, flexible -con más fluctuación que estabilidad- que se aleja de las perspectivas positivistas en términos de historiografía musical. Una de las muestras se corresponde con la emergencia y la construcción de una sonoridad rock en Brasil a fines del sesenta y otra con el rock en la Argentina en la primera democracia.

\section{CONCEPTOS OPERATORIOS DE LA MÚSICA TRANSMITIDA EN DIRECTO}

En los programas latinoamericanos de televisión estudiados el rock estuvo presente en concursos (el festival) y en la realización musical en vivo 
(recital); también se consideraron las declaraciones testimoniales de los músicos de América Latina que hicieron en entrevistas durante o a posteriori del programa. En tales muestras se observaron las transformaciones producidas en los conceptos como género musical, música popular, rock/pop, autenticidad, cantautor, público, entre otros. Tales conceptos resultan operatorios en el campo musical porque son categorías que permiten comprender y transformar materiales mediante la articulación de reglas y a partir de una práctica disciplinar determinada. No siendo apriorísticos ni universales, estas categorías son operacionales en el área en las que son producidas y utilizadas. Estas nociones habilitan al sujeto a comprender y a modificar no solo lo que consume de la música, sino lo que construye y elabora a partir de ella, de ahí su importancia. Los conceptos operatorios no son las apreciaciones valorativas - en tanto juicios estéticos- que todo sujeto posee frente al hecho artístico. Por consecuencia, operan en los actos cotidianos de los sujetos orientando consumos, usos, identificaciones, apreciaciones y conocimientos sobre esas músicas y esos músicos.

Por ejemplo, la autenticidad, al expresar valoración identitaria a una música, o el término riff en el caso del rock, son susceptibles de comprenderse como conceptos operatorios porque admiten reconfiguraciones de las nociones de obra musical, músicos y temáticas musicales. El riff, como motivo melódico-rítmico muy breve que se reitera estableciendo la base de una composición musical (Middleton, 1999), tiene la capacidad de sintetizar la identidad de una determinada música. Se suele datar su uso en la música popular en torno al jazz en los años 1920 y se sugiere el origen del término como resultado de la abreviación de refrain, es decir, estribillo. Ese gesto habitualmente instrumental en el rock es un elemento de individualización de la canción o tema que el público rápidamente identifica sin necesidad de otro anuncio verbal. Su respuesta física, e incluso sonora, da cuenta de la comprensión musical que realiza el público. En la televisación de la realización musical, tanto la visualización del instrumento encargado del riff como la reacción del público frente a los primeros sonidos del mismo, son tomas y planos habituales en los programas estudiados. La inmediatez de la reacción del público frente a la escucha del riff, refuerza el directo y, por consiguiente, la construcción de presente temporal que el televidente posee.

\section{TELEVISIÓN MUSICAL EN DIRECTO E INDUSTRIA FONOGRÁFICA}

Si bien desde 1950 existen tanto en Argentina como en Brasil experiencias televisivas ${ }^{1}$ en las que se incluye la música como contenido, el video

1 La televisión argentina tuvo su primera emisión el 17 de octubre de 1951 cuando transmitió los discursos de Eva Perón y Juan Domingo Perón en los festejos por el Día de la Lealtad. Se trataba del original Canal 7 de Buenos Aires, dependiente de LR3 Radio Belgrano y dirigido por Jaime Yankelevich. La televisión brasileña se inició el 18 de septiembre de 1950 en San Pablo, a cargo del periodista Assis Chateaubriand, denominada TV Tupi. 
tope, el modelo comercial de explotación televisiva privada y la expansión latinoamericana de las cadenas norteamericanas CBS, NBC y ABC son factores comunes durante la década de 1960 en ambos países y en el resto de Latinoamérica (Bulla, 2008; Mastrini, 2013). Asimismo, los inicios de la televisión latinoamericana con alcances populares son contemporáneos a la incorporación y el desarrollo de formas locales de música beot y de rock and roll en la región. Sobre todo en el caso de la música denominada Nueva Ola, música iê-iê-iê o simplemente Beat, la unidad entre producción discográfica y promoción televisiva tuvo entre 1960 y 1970 gran número de producciones entre las que se conocen el Club del Clan, Alta Tensión 0 Sábados Circulares en Argentina y la Jovem Guarda en Brasil. Su inclusión en la televisión fue de la mano de programas cuyos formatos han sido copiados de los contenidos de la televisión de Estados Unidos. Durante los primeros veinte años de televisión en Brasil, se importaron tanto la programación como la música pop norteamericana, lo que expone el carácter publicitario que tuvo en ese momento dicho medio (Mattos, 1992). Por consiguiente, la industria fonográfica multinacional, los formatos televisivos especializados en música y el paulatino desarrollo del rock en la región presentan grados de interacción significativos. Alain Cohen Troussat era el presidente de la filial brasileña del sello discográfico multinacional Phillips en 1967. Zuza Homem de Mello informa en su libro La Era de los Festivales (2003), que en septiembre de 1967, en una reunión de productores fonográficos en Río de Janeiro, Troussat (en Homem de Mello, 2003) propuso:

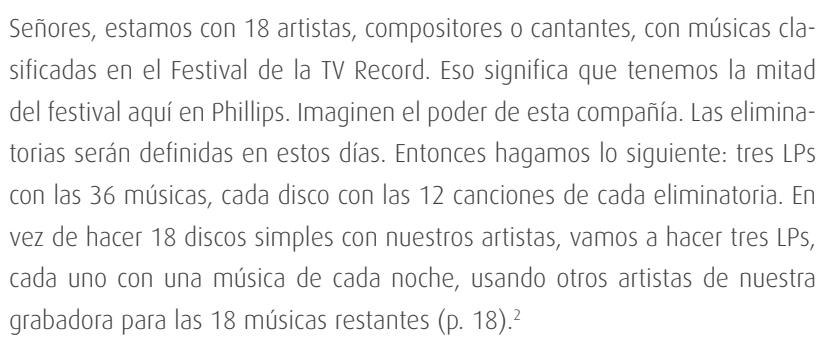

Para el 30 de septiembre, en la presentación de la ronda de finalistas del III Festival de la Música Popular Brasileña de la TV Record, ya existía uno de los tres LPs y se presentó en el propio programa. Otro hecho que da cuenta de la interacción entre industria fonográfica, promoción televisiva de la música y mercado discográfico regional es la edición en castellano

2 «Senhores, estamos com 18 artistas, compositores ou cantores, com músicas classificadas no Festival a Record. Isso significa que temos metade do Festival aqui na Philips. Imaginem o poder desta companhia. As eliminatórias serão definidas nestes dias. Então, façam o seguinte: três LPs com as 36 músicas, cada disco com as 12 de cada eliminatória. Em vez de fazermos 18 compactos simples com nossos artistas, vamos fazer três LPs, cada um com as músicas de cada noite, usando outros artistas da nossa gravadora para as 18 músicas restantes» (Troussat en Homem de Mello, 2003, p. 18). Traducción de la autora del artículo. 
de la canción «Alegría, alegría», de Caetano Veloso. En 1967 dicha canción obtiene el cuarto puesto en el mencionado Festival y es interpretada por el cantautor, acompañado de un grupo de rock argentino, los Beat Boys, además de la orquesta broodcosting del programa. En 1968 Billy Bond graba un EP titulado Yo, Billy Bond, ${ }^{3}$ en el sello argentino Music Hall. Entre los cuatro temas que lo componen se encuentra una versión en castellano de "Alegría, alegría». La traducción es del propio Bond y los arreglos corresponden a José Carli. Obviamente la letra de la canción de Veloso, como todas las canciones que integraban el Festival, había pasado por la revisión de las autoridades de la dictadura, que en el caso puntual implicaba modificaciones del original realizadas por el compositor a exigencia del autoritarismo y a los fines de evitar una censura.

Sean Stroud, Zuza Homen de Mello y Geni Rosa Duarte, entre otros, han estudiado formalmente la importancia de los festivales televisivos de música para la configuración de la música popular brasileña, así como para la inclusión masiva de ese sonido universal que la Tropicalia desea en Brasil, según palabras de Veloso y Gil. La propia Red Record de televisión brasileña volvió a homenajear ese III Festival con una retrospectiva del mismo conducida por Solano Ribeiro, el productor original, que fue emitida en 1988. El documental también revisitó su impacto buscando en testimonios directos de los protagonistas las relaciones entre música, mercado, política y televisión como es el caso de Uno noite em 67 (2009) dirigido por Renato Terra y Ricardo Calil. En todos estos casos existe un apartado para considerar el sonido de las guitarras eléctricas, y en ellas los teclados, la batería y el riff del rock que asomaba en la música popular brasileña, a través de su televisación.

La conjunción entre compositor e intérprete en una única persona identificada con la figura del cantautor ha resultado una forma de legitimación del rock como género musical, señala Keir Keightley (2006). Dicha integración de la creación musical con su performance permitió en el rock la afirmación sobre la autenticidad del género frente a otros. De esa forma, señala el autor, los músicos de rock de finales de los sesentas configuraron una manifiesta demostración de la integridad artística, incluso en términos éticos, que entronaba la figura del cantautor (Keightley, 2006). En estos casos, la unidad entre creación musical y exposición pública liga al rock a lo auténtico, a lo no tergiversado, a los comportamientos que existieron en el folk norteamericano, a lo que se instala como verdadero frente a lo falso o inauténtico. Veremos en los análisis propuestos que el rock en la región también asumió dicha integridad artística en los cantautores, figuras que pudieron componer esa música angloparlante en sus lenguas vernáculas, con medios tecnológicos internacionales adaptados a las realidades propias pero con apelaciones al ritmo y a la sonoridad local.

3 El disco está catalogado en Music Hall con el código MH 60.297, B-2.

6 Clang 


\section{YO PIENSO EN CANTAR EN LA TELEVISIÓN ${ }^{4}$}

Se llamó Passeata contra las guitarras eléctricas a la manifestación pública que reunió en San Pablo el 18 de julio de 1967 a unas 300 personas lideradas por músicos de Brasil. Embanderando la lucha contra la música foránea, principalmente identificada con el rock y particularizada a nivel sonoro en las guitarras eléctricas, asistieron a la manifestación, entre otros músicos Elis Regina, Gilberto Gil, Edu Lobo y Jair Rodríguez. Dicha acción desafiante en dictadura tuvo también repercusiones como forma de autoafirmación de la industria cultural brasileña, aunque fue también asimilada como promoción de un programa de televisión sobre la música del país continental. Así, el programa televisivo Frente única: noite do músico popular brasileira de la TV Record obtenía visibilidad ciudadana a la vez que se situaba en una manifestación popular durante la dictadura militar iniciada en 1964. Ese hecho ocurrió dos meses antes del III Festival de Música Popular Brasileña de la TV Record, una competencia televisiva musical en la cual músicos de Brasil presentaban sus canciones y un jurado de expertos así como el público de sala seleccionaban a los ganadores. En ese festival de la TV Record obtiene el segundo puesto la canción de Gilberto Gil «Domingo no parque». El arreglador del tema fue Rogelio Duprat, un compositor pionero en la música electroacústica en Brasil, formado en la música experimental de la segunda vanguardia europea, y uno de los más importantes miembros de la Tropicalía. En 1967 logró también ser premiado por su trabajo con el arreglo de «Domingo no parque» en ese festival. Esa situación lo llevó a integrar parte de un álbum que compila sus arreglos y los de Lindolpho Gaya, ambos ganadores de festivales televisivos. El álbum se llamó Os maestros premiados, ${ }^{5}$ editado por Phillips en 1968. Esto expone que tanto los músicos cantautores como los arregladores lograban popularidad mediante el programa televisivo.

«Domingo no parque» incluía en la instrumentación a la banda broadcasting de la TV Record, a Os Mutantes, con guitarra eléctrica, bajo eléctrico y platillos de entrechoque, así como un berimbao tocado por el percusionista Dirceu Xuxu Medeiros. La canción transmitida comienza con una introducción de la orquesta que realiza una sección homorrítmica a varias partes, con tempo ágil y una sonoridad de espectro muy amplio, a la que se adicionan el berimbao y la banda de rock tocando todos el pulso regular. La rítmica y la disposición melódica inicial se aproxima a la cantiga de capoeira que sigue en la canción, a la vez que nos acerca al fenómeno transcultural y mestizo de la música latinoamericana. En este caso, se intercepta con el beat y el rock angloparlante que se difundiera en el mercado brasileño entre la juventud de clase media y el público estudiantil

4 «Eu pensó em cantar na televisión», traducción de la autora del verso extraído de la canción «Alegría, Alegría», de Caetano Veloso, 1967.

5 Catalogado en su versión mono con el código R 765.029L. 
universitario vinculado con la izquierda. Este último integraba una parte importante de los asistentes a las transmisiones del festival. La primera sección musical supone dos grandes partes que se dividen armónica y melódicamente, por el tempo y los arreglos vocales. La primera parte, más lenta que la introducción, posee un bajo melódico con instrumentos de viento y bajo eléctrico. La guitarra acústica de Gilberto Gil realiza un ostinato que marca el contratiempo. La voz principal es alternada con el coro que durante esa estrofa interviene con un pequeño relleno melódico al unísono en salto ascendente de tercera. Una transición orquestal media hacia la sección siguiente, donde la melodía principal se hace más continua y solo en los dos últimos versos interviene el coro. Los vientos metal rematan repitiendo la misma frase de salto de tercera que el coro hizo en la primera parte. La segunda sección, contrastante, se caracteriza por la inclusión de mayor tensión armónica, por la yuxtaposición tonal como procedimiento modulatorio, y por el ascenso tonal y el aumento paulatino de la intensidad sonora. Al asociar dicho crecimiento general de la complejidad armónica y sonora al desenlace dramático de la narrativa poética de la canción, la tensión general del tema implica una mayor densidad cronométrica en las contramelodías de las cuerdas frotadas. Un retorno final a la primera sección está marcado por el cambio de tempo, rallentando el ritmo total en el desenlace final narrativo. El berimbao está presente en todo el tema y suena con patrones propios de la danza capoeira. Excepcionalmente en la segunda sección los platillos de entre choque no suenan, el resto del tema están asociados al contratiempo y a la unidad tímbrica con el berimbao.

Como en todos los casos del III Festival en 1967 existió un backstoge permanente en la transmisión, una cámara a la izquierda del espectador mostraba alternando con la cámara frontal, la espera, las conversaciones y los gestos de los músicos segundos antes de salir a escena. En la presentación de «Domingo no Parque», quien está en cuadro es Gilberto Gil que, con actitud dubitativa, intenta ingresar no bien el locutor indica el tema, pero lo anunciado es el ingreso de Os Mutantes como la banda acompañante y Gil es retenido por los asistentes del programa fuera del escenario. La visualización de estas situaciones resulta de importancia al momento de considerar la reformulación conceptual que puede realizarse mediante la televisión formal ya que

[...] la semejanza entre el concierto o el teatro visto en la sala original, o visto desde la pantalla, se acentúa en los estrenos, cuando lo que se retransmite no son únicamente las actuaciones del escenario, sino también las ceremonias preambulares de los vestíbulos, o las intervenciones del público en la sala original, mediante sus aplausos o sus abucheos (Bueno, 2000, p. 224).

Los primeros planos laterales del músico se visualizan aún mientras acomoda el banco sobre el que se apoyará para tocar la guitarra. La introducción 
es mostrada en un plano panorámico fijo y estático, como es el ostinato musical que se escucha. Luego de la introducción instrumental se observa a Gil en un plano detalle en el que indica el ingreso de la melodía principal con un gesto al resto de los músicos. La proximidad de la toma permite al televidente conocer, incluso, las gestualidades entre los músicos. Esa toma se alterna con un plano corto de Arnaldo Baptista, el bajista de Os Mutantes, que hace el coro. Ese procedimiento se mantendrá en el tiempo enfocando en forma alterna a Rita Lee y Sérgio Dias con primeros planos, a los que se suman planos panorámicos del escenario en los que el tutti orquestal con la banda coinciden en la transición musical. Un plano americano de Gil permite ver por detrás al director y al contrabajista de la orquesta, expectantes al cambio de tempo. En esta transmisión el público no aparece, sólo se escucha cuando se produce un fuera de campo que recuerda su presencia masiva en el teatro. Tampoco se muestra el jurado, pero al no emitir sonido su presencia es totalmente desapercibida. En el final, una vez terminada la actuación y aún con la banda sobre el escenario un plano panorámico que se abre permite ver a contraluz al público. El final del arreglo implica un coro con desplazamiento rítmico entre las voces. La actitud triunfal de Gilberto Gil en ese momento está expresada en la incorporación corporal que lo muestra con un brazo en alto y con el otro sosteniendo la guitarra. Esa secuencia estuvo integrada por planos cercanos a Gil y Os Mutantes, y planos panorámicos de la banda con la orquesta detrás. Dicha alternancia de planos en tiempo real está también en sincronía con el ritmo general de la canción.

Al tratarse de un concurso, y ser el tema de Gil ganador del segundo puesto, la performance del mismo se reitera una vez conocido el resultado. En esa oportunidad el público aparece en escena y se muestra el palco superior donde se pueden observar, incluso, los pósteres de Gilberto Gil que algunos asistentes tenían. En esta segunda oportunidad, la alternancia de cámaras con planos cortos sigue organizando la transmisión sólo que esta vez se usa el encuadre cenital que provoca un cambio de perspectiva sobre los músicos. La centralidad en ambas transmisiones audiovisuales estuvo en el cantautor, a quien, además, se lo muestra como organizador de las entradas, de los incrementos de intensidad y de los cambios de tempo, aun cuando hay un director de la orquesta acompañante. La figura del cantautor en este caso implica, también, la construcción de una sonoridad rock que mezcla las tradiciones de la capoeira con la experimentación armónica de la bossa. Tal como lo expone Geni Rosa Duarte (2006):

En esta composición, la tradición nacionalista (expresada en el uso del berim-

bao y del ritmo regional, capoeira) se contrapone a lo nuevo, en la descripción

de una enredo cotidiano con sonidos dispersos y ruidos, contrapuestos a la 
voz de Gil contrastando con el coro joven de los Mutantes, anticipando una

verdadera «jalea real» $($ p. 47$){ }^{6}$

Esas propuestas sonoras con guitarras eléctricas, a partir del consumo popular y de la televisación, pudieron no solo ampliar el concepto de música popular brasileña, sino, también, cuestionar el aparente nacionalismo sonoro de la bosso nova para afianzar la condición mestiza.

En el backstoge del programa, también transmitido en tiempo real, Caetano Veloso es entrevistado entre otros músicos participantes. Se le pregunta por la definición de la música pop. El cantautor de Bahía indica:

Es una cosa que yo admito como tema porque de alguna forma la gente está tendiendo a asumir una forma de cultura pop que sería todas las formas de cultura masificada, pop es en inglés una cosa del arte de masas, de lo popular, que se utiliza para todo aquello que tiene suceso de masas, que tienen elementos o cosas que conllevan comunicación directa con la masa, como tiene

la pintura pop (Veloso en Ribeiro, 1967, 01:02:46).

El desarrollo conceptual es interrumpido por el periodista, quien compara el contenido de la definición con el propio Caetano Veloso y dice: "¿qué mayor comunicación con la masa que Caetano Veloso va a tener en estos tiempos, eh? Cuando entra al escenario de repente, cuando entra para cantar, domina al público con una fuerza espectacular» (Ribeiro, 1967, 01:03:20) ${ }^{7}$

Con ese remate, el periodista centra en la figura del músico el concepto que en el desarrollo de Veloso no tenía relación con los artistas sino con la forma social de una cultura, siempre colectiva. Independientemente de desarrollos conceptuales formales sobre el término pop, es claro que entre una definición y otra existió una reformulación conceptual.

\section{EN ESA LUNA TELEVISADA ${ }^{8}$}

Armusa (Archivo musical suramericano) puso a disposición pública una de las emisiones del programa Badía y Compañía de 1984, transmitido por Canal 13, por ese entonces un canal público. Dicho programa tenía un

6 «Nessa composição, a tradição, até mesmo a tradição nacionalista (expressa no uso de berimbau e do ritmo regional, capoeira) se contrapunha ao novo, na descrição de um cotidiano entremeado com sons esparsos e ruídos, contrapostos à voz de Gil contrastando com o coro jovem dos Mutantes, antecipando uma verdadeira "geléia geral"» (Duarte, 2006, p. 47). Traducción de la autora del artículo.

7 «É uma coisa que eu admito como um assunto porque de alguma forma as pessoas estão tendendo a assumir uma forma de cultura pop que seria todas as formas de cultura de massa, pop é em inglês uma coisa de arte de massa, do popular, que é usado para tudo o que tem eventos de massa, que têm elementos ou coisas que envolvem comunicação direta com as massas, como a pintura pop». (Veloso en Ribeiro, 1967, 01:03:20) Traducción de la autora del artículo. 8 Verso extraído de la canción «Del 63» (1984), de Fito Paéz. 
recital en vivo al final del mismo. En este caso el recital fue de Fito Páez, quien interpretaba sus propios temas, tocando piano y teclado, junto a Fabián Gallardo en guitarra y coros, Paul Rouge en bajo y teclado, Tweety González en sintetizador y teclado, y Daniel Wirtz en batería. Entre los temas que se incluyen en el recital, la canción «Del 63» implica un cambio para la transmisión en directo. La canción titula el primer disco de estudio del cantautor rosarino, editado por Emi Odeón S. A. en 1984. ${ }^{9}$ El tema presenta tres secciones diferenciables en melodía, armonía e instrumentación. La primera sección posee una estructura melódica organizada a partir del salto ascendente de sexta mayor seguida de un arpegio descendente interrumpido en una cuarta aumentada respecto de la tónica principal. Ese motivo se reitera transportado con similar ritmo y completa la frase un consecuente de menor duración, asentado sobre un sexto grado descendido. La segunda sección alterna armónicamente entre la subdominante y la tónica en la primera frase, durante tres versos que culminan en una cadencia sobre tónica.

Fito Páez es presentado en primer plano tocando el piano y cantando «Del 63». Antes de terminar la primera frase invita al público a sumar su voz dado que «no hay nada acá» (Páez, 1984, 00:20:12), haciendo referencia al único acompañamiento de piano con el que por entonces cuenta la performonce. Cuando el público se suma, el cantautor indica al final de la primera estrofa que los integrantes del público «están más afinados que yo» (Páez, 1984, 00:20:20) en alusión a las condiciones vocales del propio músico, que pueden, incluso, escucharse en el resto del recital, pero también a las condiciones de la melodía de la primera estrofa. El rango melódico de las dos estrofas de la primera sección es de séptima menor, aumentando hasta una octava en la segunda sección. Dicha extensión es abordada fundamentalmente mediante grado conjunto y bordaduras, excepto por los inicios en anacrusa donde predomina el salto de sexta, o en los arpegios descendentes por terceras. Si bien, los cromatismos existentes son eventuales todos responden a las condiciones del ciclo armónico de la canción.

En la tercera estrofa, se incorpora el sintetizador con timbre de cuerdas frotadas, tocado por Tweety González. En este caso, el ciclo armónico se desarrolla sobre un bajo con la tónica como nota pedal. Para la tercera sección de la canción, cuyo carácter es más lírico, el público tiene su primera intervención a solo, sin Páez cantando aunque él duplicaba parte de la melodía en el registro agudo del piano y adornaba las notas largas con movimiento por grado conjunto. Esta es la primera modificación importante respecto de la versión de disco del mismo tema. La transmisión televisiva de esa situación alterna entre un plano medio y otro general del público

9 Número de catálogo del LP: 8142406518214. 
cantando. Los tres primeros versos están elaborados sobre el acorde de tónica con séptima mayor. Fito Páez anuncia al público y también al resto de la banda que se quedará solo cantando, mediante un gesto. Esa situación se muestra en un plano general picado donde el público a contraluz se ubica en la base inferior de la pantalla y la banda iluminada de frente. Para la segunda frase la escena tiene un plano medio más cercano, de frente al público, el cual oscila al compás y canta. En esa situación, los conductores del programa, Juan Alberto Badía y Silvia Fernández Barrios, se tuercen para ver la interpretación en vivo del público dando la espalda a la cámara, lo que refuerza la situación del directo. La visualización de los conductores como espectadores era común sólo en los recitales de cierre del programa, que también tenían otro estudio como locación dado el mayor número de público que albergaban. Dicho estudio tenía sus propios técnicos de sonido e imagen, lo que se explicita en los títulos finales del programa.

En las sucesivas estrofas la banda acentúa frases específicas mediante su acción sonora, como cuando dice "no se puede andar sólo en la calle, sin un revolver» (Páez, 1984), en la cual la batería, la guitarra y el bajo suenan a gran intensidad. Con la reiteración de la tercera sección el público canta a capella y es posible observar a Páez indicarle a González que deje de tocar el acompañamiento. Esta vez la cámara frente al público muestra una panorámica del mismo al que también se acerca en un zoom y reencuadra hacia el último verso de la sección. En lugar de volver a la sección uno, Páez retoma la segunda sección con una única estrofa, en la que al final se produce una transposición tonal ascendente e ingresa el resto de la banda en forma constante. La transmisión muestra el gesto de Páez hacia el acento en el cual la banda ingresa luego de un trémolo de Toms en la batería. La intensidad y sonoridad del tema en general crece y se amplía en espectro, lo que anticipa el final donde, mediante un descenso de velocidad e intensidad, se completa el ciclo. Los gestos internos a la ejecución, la espontaneidad del coro que integró el público, los comentarios alusivos a la performance por parte de Fito Páez, las risas cómplices de los conductores, así como las pequeñas ausencias melódicas o de acompañamiento producto de las decisiones e indicaciones dadas, exponen el directo.

Se trata, entonces, de ese vivo, de la sonoridad del momento que, aún mediada por la transmisión, acerca al televidente a los sucesos escénicos televisados y a la experiencia del público en el estudio. Es el dramatismo que el directo incorpora, donde el imprevisto determina la condición verdadera de la televisión formal. Esa condición causa los efectos audiovisuales percibidos por los televidentes e identifica la experiencia del público en el estudio. Por ello, "este dramatismo procesual, vinculado al presente, incluye la posibilidad de una interrupción imprevista del concierto [...] y aun de una reacción directa o indirecta del espectador sobre el escenario» (Bueno, 2000, p. 224). Ese coro a capella del público puede ser también el del televidente, incluso como la claque que colabora en su palmeo incesante con la 
realización de la verdad transmitida, la participación cantada del auditorio aumenta la similitud experiencial de quien es televidente.

El rock local, como cualquier otra música, es también reformulado por la transmisión televisiva en directo, a la vez que utiliza un medio masivo para su propia afirmación inicial o su expansión a posteriori. El video clip ha sido uno de los formatos audiovisuales más asociados al rock y a su difusión por televisión, sobre todo con la emergencia de los canales temáticos a finales de 1980. Sin embargo, parte de su construcción como género local también ha sido reformulado en la televisión en directo al interrelacionar masividad, mercado fonográfico e identidad cultural.

\section{REFERENCIAS}

Bond, B. (1968). Yo, Billy Bond [EP]. Buenos Aires, Argentina: Music Hall.

Bourdon J. (1997). El directo: una política de la voz o la televisión como promesa incumplida. Revisto Réseoux, 15(81), 61-78.

Bueno, G. (2000). Televisión: Apariencio y Verdad. Barcelona, España: Gedisa.

Bulla, G. (2008). Televisión argentina en los 60: la consolidación de un modelo de largo alcance. En G. N. Mastrini (Ed.), Mucho Ruido y pocos leyes. Economía y políticas de comunicación en lo Argentina (pp. 113-134). Buenos Aires, Argentina: La Crujía.

Duprat, R. y Gaya, L. (1968). Os maestros premiodos [LP], San Pablo, Brasil: Phillips.

Gil, G. (1968). Domingo no parque. En Gilberto Gil [LP], San Pablo, Brasil: Phillips.

Homem de Mello, Z. (2003). A Era dos festivais. Uma Parábola. San Pablo, Brasil: Editora 34.

Keightley, K. [2001] (2006). Reconsiderar el rock. En S. Frith y otros (Comps.), La otra historia del Rock. Aspectos clave del desarrollo de la música popular: desde las nuevas tecnologías hasta la política y la globalización (pp. 155-194). Barcelona, España: Ediciones Robinbook.

Mastrini, G. N. (2013). Las industrios culturales en Argentina (Tesis de doctorado). Departamento de periodismo III, Universidad Complutense de Madrid, Madrid, España.

Mattos, S. (1992). Un perfil de la televisión brasileña: 40 años de historia (1950-1990). Comunicación y sociedad, (16-17), 45-74.

Middleton, R. (1999). Form. En B. Horner y T. Swiss (Eds.), Key terms in Popular Music and Culture (pp. 146-148). Oxford, Inglaterra: Wiley-Blackwell.

Páez, F. (1984). Del 63. En Del 63 [CD]. Buenos Aires, Argentina: Emi Music. Pérez Ornia, J. R. (2011). ¿Qué es la televisión? En J. La Ferla (Comp.), Televisiones. Coloquio internacional sobre TV (pp. 17- 39), Ciudad Autónoma de 
Buenos Aires, Argentina: Fundación Telefónica.

Ribeiro, S. (1967). A grande final Festival TV Record [DVD], Brasil: TV Record. Duarte, G. R. (2006). Acordes precisos e discursos dissonantes: debates estéticos e políticos em torno da Tropicália. En TEMAS \& MATIZES, (10), 46-52. Shuker, R. [1994] (2001). Understonding Popular Music. Londres, Inglaterra: Routledge.

Terra, R. y Calil, R. (2010). Uno noite em 67 [Documental]. Brasil: Video Filmes.

Veloso, C. (1967). Alegría [EP]. Brasil: Philips. 